\title{
Frequency of delusional infestation by proxy and double delusional infestation in veterinary practice: observational study
}

\author{
Peter Lepping, Mark Rishniw and Roland W. Freudenmann
}

\section{Background}

Nothing is known about the prevalence of delusional infestation in veterinary practice and the consequences for psychiatrists.

\section{Aims \\ We attempted to examine the frequency of delusional infestation among pet owners presenting their animals to veterinary clinics.}

\section{Method}

We conducted a survey among 32663 veterinary clinicians who were members of the Veterinary Information Network.

\section{Results}

The respondents had seen 724 suspected cases of delusional infestation by proxy in a pet. The clients were mainly White, female and 30-60 years old. They presented mainly dogs and cats, and the alleged infestation was mainly with arthropods or worms. Also, 252 clients claimed to be affected themselves; we termed this 'double delusional infestation'

\section{Conclusions}

Delusional infestation is seen frequently in veterinary practice. Psychiatrists need to be aware that patients may have pets they believe are infested.

\section{Declaration of interest}

None.
Delusional infestation is a psychiatric disorder that manifests as an unshakeable false belief of being infested with living or inanimate pathogens. The chief complaint of patients is a rigid belief, against all medical evidence, that they are infested. This is usually associated with abnormal sensations that the patients attribute to the alleged infestation. ${ }^{1}$ Since the condition was first described in 1894 more than a dozen different names have been applied to the disorder, most commonly delusions of (or delusional) parasitosis and Ekbom syndrome. ${ }^{1}$ Approximately $40 \%$ of patients present with a primary monodelusional disorder. ${ }^{2,3}$ The remaining presentations occur as a secondary delusional infestation in the context of other illnesses, most commonly neurological or psychiatric problems or as a side-effect of medication (e.g. secondary to cardiovascular accidents, dementia, schizophrenia, substance misuse, diabetes or antibiotic therapy). ${ }^{4}$ Both primary and secondary delusional infestation can present as a monodelusional disorder, a shared delusion or a delusion by proxy. In monodelusional disorder only the patient is involved and it is only the patient who describes symptoms. In shared delusions the patient as the primary inducer, as well as someone in his or her immediate vicinity, share the belief that an infestation is present. In delusional infestation by proxy patients claim that someone other than themselves is infested; this may be the patient's child, spouse or pet.

Delusional infestation by proxy or as a shared delusion involving pets has been poorly described in the literature, and is limited to case reports or brief discussions in newsletters. ${ }^{5-8}$ These case reports usually describe pet owners who present a cat or dog to a veterinary clinician with an unshakeable but false belief that their pet is infested, commonly with a living object or parasite. They may present the veterinary clinician with a specimen sign (formerly known as the 'matchbox' sign) - an example of the alleged pathogen which they have collected from the pet. ${ }^{1,9,10}$ They may or may not exhibit signs of, or profess to, infestation. They may undertake efforts to eradicate the infestation using various ectoparasiticides on their pets that could result in chemically induced pathology. Owing to the paucity of research in this field it is completely unclear as to how common such presentations are in veterinary care. In addition, it is unclear how many cases are delusions by proxy and how many are 'shared' delusions where the pet owners believe that both they and their pet are infested. It is difficult to call this a shared delusion because the pet is unable to share the delusional belief of the owner, but is merely the innocent victim in the psychosis. We therefore suggest the new term 'double delusional infestation' for patients who believe that they and someone or something else is infested, but the other person or pet does not or cannot share that belief.

Finally, patients with delusional infestation by proxy with alleged infestation of their pet might not present to a psychiatrist for evaluation, and they are unlikely to be seen by a physician who would make such a referral. Instead, they are likely to seek advice from a veterinarian, who is unable to recommend or make a referral to a mental health specialist. Thus, this variant of delusional infestation by proxy is understandably poorly recognised in the field of psychiatry. We therefore attempted to examine the frequency of delusional infestation among pet owners presenting their animals to veterinary clinics.

\section{Method}

We developed a questionnaire that was emailed to 32663 veterinary clinicians primarily working in first-opinion practice in the USA and Canada who were members of the Veterinary Information Network (www.vin.com), a subscription-based worldwide online community and information service for veterinarians (the full questionnaire is available as an online supplement to this paper). Data were collected from 23 July 2013 to 21 September 2013. The questionnaire asked all participants whether they had ever seen pet owners (referred to here as 'clients') whom they suspected had delusional infestation by proxy. We asked for a demographic profile of the clients as well as the type of pet they presented as 
allegedly infested. We enquired what diagnostic tests were performed to examine for infestation, whether any infestation was found, whether the pet exhibited lesions, what the veterinary clinician explained to the client and whether treatment was suggested for the pet. We also asked whether requests were made to euthanise the animal and what kind of action, if any, the veterinarian may have initiated when delusional infestation was suspected. In a follow-up questionnaire to participants who had seen at least one case of suspected delusional infestation, we asked whether the client described symptoms himself or herself or exhibited clinical signs such as excoriations on the face, hands or arms, consistent with scratching, and whether any examination was performed to confirm or dismiss such symptoms.

\section{Results}

We had a total of 714 responses from the 32663 email requests for participation with sufficient data for analysis. The return rate for our questionnaire is in keeping with the return rate of other surveys in this professional group performed through the network. The 714 respondents had seen 724 suspected cases of delusional infestation. Table 1 describes the location and type of the participating clinics. Most respondents were first-opinion practice clinicians from the USA or Canada. Table 2 describes the demographic characteristics of the clients: most were White women $(n=566 ; 78 \%)$; of these $72 \%$ were aged $30-60$ years. Table 3 describes the type of animal presented to the veterinarian with the alleged infestation, and Table 4 describes the alleged infesting pathogen that the client identified on their pet. Most were purported to have ectoparasites. Clients identified 'lesions' in 483 cases; however, veterinary clinicians identified lesions on only 94 of the pets; of these, 88 were claimed by the clients to be the result of the infesting pathogen. Table 5 summarises the answers to all additional questions.

Of the 307 cases in which the veterinary clinician tried to take action regarding the client's probable diagnosis, 209 veterinarians recommended a visit to the client's physician, 88 spoke to a family member and 145 took other forms of action, including recommendations to fumigate the house, accompanying the client to a physician for a consultation, visiting the client's home to ensure that there were no parasites in the house, or offering to discuss the situation with the client's physician. Several clients or their relatives told the veterinary clinician that they were under the care of a mental health professional for delusional infestation or other mental illness (e.g. schizophrenia). From the 252 clients who claimed to be affected, 147 demonstrated alleged cutaneous lesions to the veterinary clinician. In 114 of these cases the clinician confirmed that a lesion was present, but in 33 clients no lesion was evident to the clinician. The clinician identified lesions in 13 clients who claimed to be infested but did not report lesions, and in 7 clients who did not claim to be infested. In 56 cases clinicians did not look for evidence of delusional infestation in the client.

\section{Discussion}

This is the first study worldwide looking into delusional infestation in veterinary practice. Its findings suggest that delusional infestation by proxy or double delusional infestation is more common in veterinary practice than previously believed. In the overwhelming majority of these cases the veterinary clinicians were unable to find any evidence of such an infestation despite reasonable attempts to do so. Even if we assume that all those who did not respond to the questionnaire had never seen

\begin{tabular}{|c|c|}
\hline & Number (\%) \\
\hline \multicolumn{2}{|l|}{ Location of clinic } \\
\hline USA or Canada & $664(93)$ \\
\hline South or Central America & $0(0)$ \\
\hline Europe & $17(2)$ \\
\hline Australia or New Zealand & $28(4)$ \\
\hline Asia or Middle East & $4(1)$ \\
\hline Africa & $1(0)$ \\
\hline \multicolumn{2}{|l|}{ Clinic type } \\
\hline First-opinion practice & $597(84)$ \\
\hline Emergency practice & $40(6)$ \\
\hline Mobile practice & $4(1)$ \\
\hline Relief veterinary clinic & $11(1)$ \\
\hline Referral practice & $39(5)$ \\
\hline Clinical academia & $23(3)$ \\
\hline
\end{tabular}

\begin{tabular}{|lc|}
\hline Table 2 Client demographic characteristics \\
\hline \\
Gender \\
Male \\
Female \\
Do not recall \\
\hline Ethnicity \\
White & $117(16)$ \\
Black or African American & $599(83)$ \\
Hispanic or Latino & $8(1)$ \\
Asian & \\
Native American or Alaskan Native & $678(94)$ \\
Hawaiian & $5(1)$ \\
Mixed race (unspecified) & $10(1)$ \\
Unknown & $4(0)$ \\
Prefer not to answer & $1(0)$ \\
\hline Age, years (estimated) & $0(0)$ \\
<20 & $3(0)$ \\
20-30 & $22(3)$ \\
30-40 & $1(0)$ \\
40-50 & \\
$50-60$ & $3(0)$ \\
60-70 & $17(2)$ \\
>70 & $90(12)$ \\
Not reported & $260(36)$ \\
\hline Economic background & $196(27)$ \\
Affluent or wealthy & $82(11)$ \\
Upper-middle class & $63(9)$ \\
Lower-middle class & $13(2)$ \\
Working class & \\
Working poor & $25(3)$ \\
Poor or impoverished & $329(45)$ \\
Not reported & $212(29)$ \\
& $135(19)$ \\
& $15(2)$ \\
& $7(1)$ \\
& $1(0)$ \\
\hline
\end{tabular}

a case of delusional infestation, and exclude the 25 cases in which an actual infestation of the animal was found, we still have a very conservative estimate of $2.3 \%$ of veterinary clinicians approached having seen at least one case. Thus, this condition is common enough to warrant further investigation and provision of guidelines for veterinary clinicians in dealing with such clients. Previously, studies have identified a predominance of elderly women with delusional infestation. ${ }^{1,2}$ In our findings the majority of clients were women, but there was a wide distribution of age and social class with only a mild bias towards people who were more able to afford veterinary services. This could, in part, reflect the typical demographic profile of veterinary clients in the USA or Canada, and a potential decrease in pet ownership among the elderly.

Encouragingly, virtually no responding clinician ignored or dismissed the client's claims without investigation. Most clinicians 


\section{Table 3 Type of animal presented}

\begin{tabular}{|lc|}
\hline Dog & $n(\%)$ \\
\hline Cat & $472(65)$ \\
\hline Bird & $199(27)$ \\
\hline Small mammal & $27(4)$ \\
\hline Fish & $6(1)$ \\
\hline Reptile or amphibian & $0(0)$ \\
\hline Small ruminant & $1(0)$ \\
\hline Large ruminant & $2(0)$ \\
\hline Horse & $0(0)$ \\
\hline Other & $1(0)$ \\
\hline Not reported & $11(2)$ \\
\hline
\end{tabular}

\begin{tabular}{|lc|}
\hline Table 4 & Pathogens alleged by clients to be infesting their pet \\
\hline Arthropod (fleas, ticks, mites, etc.) & $n(\%)$ \\
\hline Bacterium & $481(66)$ \\
\hline Worm & $3(0)$ \\
\hline Not specified & $156(22)$ \\
\hline Other & $21(3)$ \\
\hline Not reported & $56(8)$ \\
\hline
\end{tabular}

performed various diagnostic tests, as mandated by the pet species, financial limitations of the client and alleged type of pathogen. Similarly encouraging was the observation that half the respondents either attempted to suggest that the client consult a physician, or talked to family members about their concerns for the client. Less encouraging is the fact that the vast majority of veterinary clinicians treated the animal with what they considered to be innocuous treatment, often using the excuse of 'just in case I missed something', or because 'the pet could use a flea control product (or de-wormer, or anti-tick product) anyway'. However, this type of action reinforces the delusional belief of the client. We understand the ethical and medical dilemmas as well as the financial considerations for privately practising veterinary clinicians who do not want to leave a client disappointed, or believe that if they 'successfully' treat the pet's infestation the situation will resolve and the client's delusion will disappear.
However, in accordance with our general advice in delusional infestation, ${ }^{11}$ it always seems appropriate to avoid any action that might reinforce the client's delusion, such as futile treatment.

Our study also revealed that approximately a third of clients identified symptoms not only in their animals but also in themselves. Equally worrying is the fact that approximately half of the clients had already treated their animals with pesticides of some description at the time of presentation. It makes it likely that they might have treated themselves as well with equally inappropriate treatments. This points to the possible use of potentially harmful substances on animals and possibly the pet owners themselves, which is in keeping with our previous research into delusional infestation. ${ }^{1}$ Given that the animal cannot consent to such treatment it brings up ethical issues regarding the responsibility of the veterinarian to avoid any action that might support the client's delusional belief. It is encouraging that veterinary clinicians appear openly to want to help their clients with these difficulties, and we have produced specific guidelines for veterinarians in line with our general recommendations. ${ }^{11,12}$

About half of clients who reported lesions on themselves had these verified by the veterinary clinicians who examined them. We cannot make definite statements on the nature of these lesions for the reasons given above. However, in delusional infestation secondary itching is common, and many lesions exist because of itch-induced scratching, skin-picking and other forms of manipulation of the skin. Importantly, such lesions are evident only in areas that the patient can reach - characteristically, the middle of the back is spared of any lesions, because the client cannot scratch that area. Several clinicians commented that they were aware of this, and asked to examine the client's back, confirming that the distribution of lesions on the client was consistent with delusional infestation. These clients may also develop secondary super-infections which in turn reinforce the delusional belief system. ${ }^{1}$ Interestingly, only $25(3 \%)$ of the animals presented had any identifiable organism, which in each individual case might or might not have been responsible for the lesions found on the animal.

\section{Nomenclature}

From a wider terminological perspective the involvement of pets in delusional infestation has some interesting psychopathological implications. Depending on whom the client believes to be infested we can formally distinguish the constellations listed below.

\begin{tabular}{|lcc|}
\hline Table $\mathbf{5}$ Additional results and findings & & \\
& Yes, $n$ & No, $n$ \\
& & 232 \\
\hline Lesions identified by client on pet & 483 & 625 \\
\hline Lesions identified by veterinarian & 94 & 224 \\
\hline Pet treated for infestation by client & 365 & 253 \\
\hline Specimen sign & 441 & 682 \\
\hline Organism identified on pet & 25 & 688 \\
\hline Euthanasia demanded & 20 & 382 \\
\hline Pet treated by veterinarian & 322 & 33 \\
\hline Negative findings explained to client & 658 & 359 \\
\hline Additional action taken to resolve apparent infestation & 335 & 184 \\
\hline Resolution of problem achieved & 190 & 344 \\
\hline Action taken to assist client with probable delusional infestation & 307 & 83 \\
\hline Client claimed to be affected as well & 252 & 96 \\
\hline Client presented cutaneous lesions to veterinarian & 156 & 145 \\
\hline Lesions verified or identified by veterinarian & 135 & 327 \\
\hline a. Missing data in some questionnaires explain why not all rows add up to 724. & & \\
\hline
\end{tabular}


(a) If the client believes that only the pet is infested (not the client himself or herself and no close relative), we have a situation similar to Münchhausen syndrome by proxy (factitious disorder by proxy), in our case delusional infestation by proxy in a pet, where an animal rather than a child is presented to the physician as a surrogate.

(b) If the pet and the client's child or children are believed to be infested, we have delusional infestation by proxy in child and pet.

(c) If the pet owner believes that not only the pet but also he or she is infested, we have a constellation similar to a shared psychotic disorder, a concept peculiarly dropped in DSM-5. In shared delusional infestation, one person (the inducer) usually makes other close relatives believe themselves to be infested as well. The fact that animals cannot, by definition, share a delusion with their owner poses an interesting terminological dilemma. The constellation cannot be properly termed a shared or induced delusional disorder (folie à deux) because it is impossible to induce a shared belief in an animal. We therefore suggest the term 'double delusional infestation' for cases where the inducer has the delusional belief of being infested and at the same time believes that his or her animal is infested, too. The same term should be used when small children are involved who cannot, because of their young age, share a delusional belief.

\section{Study implications}

It appears that delusional infestation is a significant problem in veterinary practice that merits further study. Veterinarians should avoid any action that might reaffirm clients' delusional beliefs. A small minority of clients request the euthanasia of healthy animals which ought to be resisted. Psychiatrists and other physicians need to be alerted to the possibility that patients may present with delusional infestation by proxy in a pet and ought to ask patients in whom they suspect delusional infestation whether they have pets and believe that they are infested.

\section{Acknowledgements}

We thank Edie Lau and Nancy Hinkle for their contribution to our discussions at the beginning of the study.
Peter Lepping, MRCPsych, MSc, Centre for Mental Health and Society, Bangor University, UK, and Mysore Medical College and Research Institute, India; Mark Rishniw, BVSC, MS, PhD, DACVIM, Veterinary Information Network, Davis, California, USA; Roland W. Freudenmann, MD, Department of Psychiatry and Psychotherapy, University of UIm, Germany

Correspondence: Professor Peter Lepping, Central Wrexham Community Mental Health Team, Ty Derbyn, Wrexham Maelor Hospital, Croesnewydd Road, Wrexham LL13 7TD, UK. Email: peter.lepping@wales.nhs.uk

First received 7 Jan 2014, final revision 28 Jul 2014, accepted 6 Aug 2014

\section{References}

1 Freudenmann RW, Lepping P. Delusional infestation. Clin Microbiol Rev 2009; 22: $690-732$.

2 Trabert W. Epidemiology of delusional ectoparasitic infestation [in German]. Nervenarzt 1991; 62: 165-9.

3 Lepping P, Russell I, Freudenmann RW. Antipsychotic treatment of delusional parasitosis: systematic review. Br J Psychiatry 2007; 191 198-205

4 Lepping $\mathrm{P}$, Freudenmann RW, Huber M. Delusional infestation. In Practical Psychodermatology (eds A Bewley, RE Taylor, J Reichenberg, M Magid): 117-26. Wiley, 2014

5 Nel M, Schoeman JP, Lobetti RG. Delusions of parasitosis in clients presenting pets for veterinary care. J S Afr Vet Assoc 2001; 72 : $167-9$.

6 Lau E. When people's delusions affect pets. VIN News Service, 6 November 2013 (http://news.vin.com).

7 Lyons ET. Delusional parasitosis. ASP Newsletter 2013; 35, 7.

8 Tapp T, Mofid M. Take the Compendium challenge - case presentation. Compend Contin Educ Practicing Vet 1998; 20: 433-9.

9 Freudenmann RW, Lepping P, Huber M, Dieckmann S, Bauer-Dubau K, Ignatius $\mathrm{R}$, et al. Delusional infestation and the specimen sign: a European multicentre study in 148 consecutive cases. Br J Dermatol 2012; 167: 247-51.

10 Freudenmann RW, Kolle M, Schonfeldt-Lecuona C, Dieckmann S, Harth W, Lepping P. Delusional parasitosis and the matchbox sign revisited: the international perspective. Acta Derm Venereol 2010; 90 : 517-9.

11 Lepping P, Baker C, Freudenmann RW. Delusional infestation in dermatology in the UK: prevalence, treatment strategies, and feasibility of a randomized controlled trial. Clin Exp Dermatol 2010; 35: 841-4.

12 Rishniw M, Lepping P, Freudenmann RW. Delusional infestation by proxy what should veterinarians do? Can Vet J 2014; 55: 887-91. 